\title{
METHODS FOR ULTRASOUND SCREENING OF CARDIOVASCULAR DISEASES IN TYPE 2 DIABETIC PATIENTS
}

\author{
A. Kundurdzhiev ${ }^{1}$, A. Markova² \\ ${ }^{1}$ Clinic of Nephrology, University Hospital "Sv. Ivan Rilski", Department of Internal Medicine, \\ Medical University - Sofia, Bulgaria \\ ${ }^{2}$ Department of Internal Medicine, Medical University - Sofia, Bulgaria
}

\begin{abstract}
Diabetes mellitus is a chronic disorder that affects predominantly the carbohydrate metabolism, but also the biotransformation of proteins and fat. Many intra- and extracellular metabolic mechanisms are impaired which leads to structural changes in the vascular wall and the heart muscle. This eventually causes their functional deterioration and the end result is clinical manifestation of macrovascular incidents or heart failure. People with perturbations of the glucose metabolism (impaired fasting glucose, impaired glucose tolerance and insulin resistance) are also with a higher risk of cardiovascular diseases, even before the diagnosis of diabetes. Nowadays we have a cheap, easy and non-invasive method for early diagnosis of cardiovascular disorders, way before their clinical manifestation, and that is the ultrasound methodology. The echocardiography is a valuable technique for the detection of changes in the myocardial structure and its contractility. The tissue Doppler ultrasound is a more precise method that can detect the slightest aberrations in the heart muscle function, that could not be seen with the conventional echocardiography. Subclinical atherosclerotic changes can be determined with a Doppler scan of the big arteries (carotids, renal arteries), and the subsequent calculation of their resistive index and of the intima-media thickness. There are a lot of studies in this field which show that the structural and functional impairment could be diagnosed in diabetic patients without any complaints and with otherwise healthy hearts. This means that these diagnostic methods should be used in the routine clinical examination of every diabetic individual in order to predict and possibly prevent major cardiovascular events and severe heart failure.
\end{abstract}

Key words: diabetes mellitus, cardiovascular complications, transthoracic echocardiography, tissue Doppler imaging, Intima-media thickness, resistive index

Corresponding author: Alexandra Markova, MD, Clinic of Endocrinology and Metabolism, University Hospital Alexandrovska - Sofia, Department Internal Medicine, Faculty of Medicine, Medical University Sofia, 1, G. Sofiyski str., 1431 Sofia, Bulgaria, fax: +3592 9230 779, e-mail: alexdoncheva@gmail.com 
The prevalence of diabetes mellitus (DM) is reaching epidemic nature worldwide. In 2014 there were 422 million diabetic subjects with a more rapid increase in the incidence of type 2 diabetes (T2D) [1]. The mortality in this population is due mainly to cardiovascular diseases (CVD). The most common macrovascular complications are coronary heart disease, acute myocardial infarction (MI), stroke, peripheral vascular disease and congestive heart failure [1].

Undiagnosed DM, as well as other glucose metabolism disturbances, is a risk factor for CVD. According to the classification of the World Health Organization (WHO), there are two pre-diabetic states - Impaired fasting glycemia (IFG) and impaired glucose tolerance (IGT) [2]. The Diabetes Epidemiology Collaborative Analysis of Diagnostic Criteria in Europe study (DECODE) included 29,714 patients aged 3089 years who were followed for a period of 11 years. The study showed that CVD risk increased with increasing blood glucose on the second hour of the oral glucose tolerance test (OGTT), and the cardiovascular mortality risk started to increase from fasting glucose levels as low as $5.25 \mathrm{mmol} / \mathrm{l}$ - well below the diabetes range [3]. In the Glucose and Myocardial Infarction (GAMI) study, an OGTT was performed in patients with acute $\mathrm{MI}$ and without previously detected glucose metabolism disturbances. Only 33\% of the patients were normoglycemic, while $35 \%$ had IGT and 31\% - undiagnosed T2D [4]. These results indicate that macrovascular complications can occur even before the diagnosis of DM is made.

The ultrasound examination is frequently used in the everyday practice as a noninvasive method for the diagnosis of CVD. Such examination tools are the transthoracic echocardiography, ultrasound measurement of intima-media thickness (IMT) of the large arteries and plaque visualization, resistive index calculation within a Doppler scan.

Transthoracic echocardiography is the method of choice for the detection of early functional and structural changes in the myocardium. Conventional methods for the examination of the left ventricle function are the two-dimensional (2D) technique, the M-mode echocardiography and pulse wave Doppler. The systolic function can be evaluated by the calculation of the ejection fraction (EF): an EF > 55\% is considered to be normal; EF between $40 \%$ and $55 \%$ is slightly reduced, EF of $30-40 \%$ is moderately diminished and EF $<30 \%$ is severely impaired [5]. The pulse wave Doppler is used for measuring the velocities of the transmitral flow and diastolic filling [6]. The E-wave (E) stands for the early filling velocity (the period of left ventricle relaxation), and $A$-wave (A) is a measure of the late filling velocity due to the left atrial contrac- tion. In healthy individuals $E>A$ and the ratio $E / A=$ 0.7-1.3. The pulse wave Doppler results depend on many factors - changes in the blood flow volume, the heart rate, the left atrial volume pressure.

A more accurate method is the Tissue Doppler Imaging (TDI) that can measure the velocities of contraction and elongation of different segments of the heart muscle. The mitral annulus approaches the left ventricle apex during the systole and moves away during the diastole. Thus an $S^{\prime}$-wave is recorded (systolic wave), as well as E' and A'-waves (diastolic waves). $E^{\prime}$ (early diastolic velocity) measures the left ventricle relaxation (elongation), and $A^{\prime}$ (late diastolic velocity) determines the atrial contraction and late diastolic filling. The E'-wave reduction and the increase in $E / E$ ' ratio are the earliest signs of left ventricle diastolic dysfunction (LVDD) [7, 8]. Boyer et al. [9] found LVDD with the help of TDI in $63 \%$ of asymptomatic type 2 diabetes patients, while with the conventional Doppler only $46 \%$ of the individuals were determined to have dysfunction. Zahiti et al. [10] studied 300 patients, of them 150 patients non-obese with normotensive, uncomplicated T2D and 150 healthy control subjects. The conventional echocardiography showed no substantial differences in the systolic and diastolic function between the two groups. However, a lower E' velocity was detected in the T2D group with the means of TDI, and after performing a stress test, the differences between the groups became even more evident. TDI could be used routinely for the diagnosis of LVDD in patients with T2D, who are otherwise considered to have healthy hearts.

Coronary heart disease is the main cause of heart failure (HF) in type 2 diabetes patients. However, HF could be diagnosed in people with diabetes without any history of underlying ischemic heart disease, valvular disorders or hypertension. This cardiac entity is a separate disorder named diabetic cardiomyopathy (DC) [11]. DC has a multifactorial etiology, and its severity progresses in time. The main risk factors for early diastolic dysfunction are insulin resistance and hyperglycemia. The state of insulin resistance is characterized by an accelerated free fatty acid (FFA) metabolism in the myocardium which in turn is accompanied by increased oxygen consumption, reduced heart muscle efficiency and lipotoxicity [12]. The insulin deficiency can lead to chronic changes in the carbohydrate and lipid metabolism which cause diminished adenosine triphosphate (ATP) activity, reduced calcium uptake from the sarcoplasmic reticulum and intracellular accumulation of toxic FFA waste products. Eventually, these deteriorations induce ATP depletion, derangement in the calcium homeostasis and increased oxygen consumption from the myocardium which results 
in myocyte hypertrophy and apoptosis, their replacement with fibrous tissue, and finally - in impaired cardiac muscle contractility [13].

The structural changes in the heart muscle lead to deterioration of the LV function. Initially, there is no clinical manifestation, but in time the disorder progresses to overt HF. These changes could be diagnosed early in the course of the disease with the help of transthoracic echocardiography. LV diastolic dysfunction (LVDD) is the earliest stage in the development of cardiomyopathy [11]. There are two mechanisms responsible for the LVDD - impaired left ventricle relaxation and decreased elasticity of the ventricular wall which are caused by the increased collagen synthesis and fibrosis accumulation. The rigid left ventricle could not be filled with enough blood in order to improve the stroke volume [8].

The results of a 3-year follow up of type 2 diabetes patients without overt CVD, showed an increase from $49 \%$ to $67 \%$ in the LVDD incidence and the deterioration was mainly accounted for by age, the presence of retinopathy and the increase in the systolic blood pressure [14]. There is evidence that LVDD develops even before the onset of DM. In a study with 1063 participants, an inverse relationship between the HOMA index and E' was found, as well as positive correlation with E/E'. There was a progressive decrease in the LV diastolic function induced by the worsening of insulin resistance from individuals without metabolic syndrome (16.3\% LVDD), over participants with metabolic syndrome and no diabetes $(32.6 \%)$, to patients with diabetes (36.6\%) [15]. There was no significant difference in the LVDD incidence between the groups of non-diabetic individuals with the metabolic syndrome and type 2 diabetic patients [15, 16]. Furthermore, the participants with the two conditions had greater risk to develop dysfunction of both ventricles [16].

Except for LVDD, other echocardiographic changes that can be found in type 2 diabetes patients [10, 17] are left ventricle remodeling and hypertrophy [18], reduced EF [19], dilated left atrium [19], and valvular impairment [20]. These disorders could be diagnosed early in the course of the diabetes progression and often remain asymptomatic [19]. Jorgensen et al. found echocardiographic abnormalities in $49,8 \%$ of 1030 outpatients with DM2 and the prevailing alterations were LV hypertrophy, dilated left atrium and LVDD [19]. In the female group predominated LVDD, LV hypertrophy and increased LV mass, while in males the reduced EF was more common [19]. The researchers determined that neither the cardiac symptoms nor the clinical signs were effective to identify patients with echocardiographic abnormalities. These findings suggest that every diabetic patient should undergo an ultrasound examination regardless of the lack of overt symptoms [19]. De Jong et al. studied the impact of obesity, T2D, and hypertension on the left ventricle remodeling. The echocardiographic examination showed that obesity $\left(\mathrm{BMI}>30 \mathrm{~kg} / \mathrm{m}^{2}\right.$ ) per se does not cause LV alterations, but when in combination with other metabolic disorders (dyslipidemia, dysglycaemia), and in these cases the concentric LV remodeling prevailed [18]. Patients with obesity and hypertension had a 4.8-fold higher incidence of concentric remodeling, and a 2-fold higher prevalence of concentric hypertrophy. The normotensive, nonobese type 2 diabetic participants had comparable changes as the normotensive individuals with obesity and metabolic disorders (concentric remodeling in $44 \%$, concentric hypertrophy in 4\%). However, when T2D was combined with obesity, regardless of the blood pressure status, the LV hypertrophy incidence increased up to $36 \%$ which is similar to the results for hypertension/obesity co-existence. Furthermore, the prevalence of a third-degree LVDD (E' < $10 \mathrm{~cm} / \mathrm{s}^{\prime} \mathrm{E} / \mathrm{A}$ $\geq 2$; $E / E^{\prime}>14$ ) in both normotensive and hypertensive obesity/DM2 patients was greater than that detected in obese and T2D groups alone [18].

Aside from $\mathrm{HF}$, the $\mathrm{E} / \mathrm{E}$ ' ratio is also a predictor of cardiovascular events. Patients with $E / E$ ' values $>15$ have three times higher relative risk of myocardial infarction and stroke than the diabetics with an $E / E^{\prime} \leq 15$ [21]. In their study, Bloomstrand et al., determined that $E / E$ ' is a strong predictor of myocardial infarction, comparable with $\mathrm{HbA} 1 \mathrm{c}$, superior to and independent of age, sex, BMI, EF, IMT, LDL-cholesterol, aortic pulse pressure velocity, the global longitudinal strain, and co-existing hypertension or CVD [21]. Mitral regurgitation also can serve as a marker of early ventricular dysfunction and overall cardiovascular death. LV remodeling causes displacement of the mitral leaflets and deformation of the mitral annulus, which results in functional mitral regurgitation [20]. Rossi et al. found that even mild regurgitation increased 4.78-fold the risk for cardiovascular death, and patients with moderate-to-severe mitral regurgitation had 7.41-fold greater risk [20].

There is a lot of evidence that IMT could be used as a marker for the diagnosis of asymptomatic CVD [22, $23,24]$. There is data supporting the concept that carotid atherosclerosis is an indicator of systemic atherosclerosis [25]. The 2010 American Heart Association/ American College of Cardiology (AHA/ACC) guideline point out the use of carotid IMT (CIMT) and coronary calcium score (CAC) as a class lla recommendation for CVD risk assessment in asymptomatic adults who are at intermediate risk [22]. CIMT is measured between the intimal-luminal and the media-adventitial interfaces of the carotid artery wall [22, 26]. A value of cIMT $>1.5 \mathrm{~mm}$ indicates the presence of a plaque 
[26]. A Metaanalysis that included five large-scale studies which evaluated CVD risk gave contradictory results considering the predictive value of cIMT as a marker for cardiovascular events. In this analysis, the ARIC study (Atherosclerosis Risk in Communities), CHS (Cardiovascular Health Study) and the Rotterdam study showed that CIMT could be used to assess incident CVD risk. On the other hand, in the MESA study (Multi-Ethnic Study of Atherosclerosis) the researchers found that cIMT could not predict either coronary events or stroke after adjusting for the Framingham Risk Score [22]. These contradictory findings can be explained by the lack of a unified protocol for cIMT measurement. The meta-analysis concluded that cIMT of the internal carotid artery and at the carotid bulb is more useful than IMT of the common carotid artery [22]. The first two carotid sites are more prone to plaque formation because of the lower tangential flow pressure [22]. Nevertheless, the IMT assessment in these two regions is more difficult because it depends on the anatomical features of the particular patient and the investigator's experience [26], and is even more complicated in obese individuals [27].

The renal resistive index $(R R I)$ is calculated by the following formula: (peak systolic velocity - end diastolic velocity)/peak systolic velocity [PSV-EDV/ PSV], obtained with a Doppler examination of intrarenal arteries [30]. Values of 0.47-0.70 are considered normal, and the difference between the two kidneys is about $5-8 \%$ [30]. There is data that RRI is a marker of vascular resistance as a result of atherosclerosis [31]. RI measurements depend on the pulse pressure and the artery elasticity [30]. Conditions that cause deterioration in these characteristics lead to changes in $\mathrm{RRI}$ respectively. For example, in newly diagnosed T2D patients there is a much higher RRI in comparison to healthy controls and patients with hypertension. After nitroglycerin intake, the RRI reduction in the diabetic group is to a much less extent compared to the hypertensive and control groups which means that there is greater vascular damage in diabetes [32]. In T2D patients higher RRI values could be found even before the presentation of proteinuria and with both normal or increased glomerular filtration rates [33], whereas the difference between diabetes patients and healthy individuals disappears in high degree renal insufficiency (eGFR $<30 \mathrm{ml} / \mathrm{min} / 1.73$ $\mathrm{m}^{2}$ ) [30]. In their study, Maclsaac et al. found that RRI is significantly higher in type 2 diabetes patients with ultrasound data for LVDD (RI $0.72 \pm 0.06)$ in comparison to the participants with normal echocardiography (RI $0.66 \pm 0.06, p<0.001$ ) [34]. There is a positive relationship between RRI, LVDD and left ventricle filling pressure. These findings support the hypothesis that vascular and myocardial stiffening in diabetes are manifestations of common pathophysiological mechanisms [34]. RRI can be used as a marker of early renal impairment and atherosclerosis of the intrarenal arteries in people with diabetes.

The carotid $\mathrm{RI}$ is a predictor of cardiovascular morbidity and mortality, and the correlation between RI and the risk of CVD is comparable to the correlation between the carotid IMT and the risk of cardiovascular events [35, 36]. Changes in the carotid elasticity could be detected by RI calculation even in cases of seemingly normal ultrasound imaging, without any changes in IMT [36]. The Doppler-derived markers, such as the higher RI, lower peak systolic and end diastolic velocities, correlate with the incident cardiovascular risk [37]. Furthermore, there is evidence that the end diastolic velocity is an independent risk factor for CVD [37]. Kinouchi et al. found that the presence of T2D, but not the HbA1c level, is correlated with higher common carotid artery RI values [35].

In conclusion, type 2 diabetes patients are more exposed to acute cardiovascular events and heart failure, and their cardiovascular risk shows a positive correlation with the rise of the blood glucose levels. The ultrasound examination is a noninvasive, fast and easy method for direct visualization of the myocardial and vascular damages. Transthoracic echocardiography and tissue Doppler imaging help in detecting the earliest signs of myocardial structural and functional deterioration, while the measurements of IMT, the renal and carotid RI could be helpful in assessing the presence of systemic atherosclerosis. With the help of these diagnostic tools could often be found signs of CVD in asymptomatic diabetes patients, which makes them an irreplaceable aid in the screening for macrovascular complications in T2D.

\section{REFERENCES}

1. WH Organization. Diabetes. Fact Sheet N312 2014. http://www. who.int/mediacentre/factsheets/fs312/en/\%5D. Accessed Nov 2014.

2. World Health Organization (WHO) Consultation. Definition and diagnosis of diabetes and intermediate hyperglycaemia. 2006, http://www.who.int/diabetes/publications/Definition \%20 and\%20diagnosis\%20of\%20diabetes_new.pdf

3. The DECODE Study Group. Is the current definition for diabetes relevant to mortality risk from all causes and cardiovascular and noncardiovascular diseases? Diabetes Care 2003;26:688-696.

4. Norhammar A, Tenerz A, Nilsson G, et al . Glucose metabolism in patients with acute myocardial infarction and no previous diagnosis of diabetes mellitus. A prospective study. Lancet 2002; 359: 2140-2144.

5. Lang R, Biering M, Devereux RB et al. Recommendations for Chamber Quantification: A Report from the American Society of Echocardiography's Guidelines and Standards Committee and the Chamber Quantification Writing Group, Developed in Conjuction with the European Association of Echocardiography, a Branch of the European Society of Cardiology. J Am Soc Echocardiogr 2005; 18(12): 1440-1463. 
6. Popescu BA, Carmen CB, Carmen G. Echocardiographic assessment of diastolic heart failure. European Cardiology 2010, 6(3): 13-17.

7. Liu JH, Chen Y, Yuen M et al. Incremental prognostic value of global longitudinal strain in patients with type 2 diabetes mellitus. Cardiovasc Diabetol. 2016;15:22. doi: 10.1186/s12933016-0333-5.

8. Mitrovska S, Lazeska B. Contemporary Echocardiographic Techniques in Early Detection of Diabetic Cardiomyopathy. J Cardiol Curr Res 2014; 1(5): 00025.

9. Boyer J, Thanigaraj S, Schechtman KB, Perez JE (2004) Prevalence of ventricular diastolic dysfunction in asymptomatic, normotensive patients with diabetes mellitus. Am J Cardiol 93(7): 870-875.

10. Zahiti BF, Gorani DR, Gashi FB, et al. Left Ventricular Diastolic Dysfunction in Asymptomatic Type 2 Diabetic Patients: Detection and Evaluation by Tissue Doppler Imaging. Acta Informatica Medica 2013;21(2):120-123.

11. Hölscher ME, Bode C, Bugger H. Diabetic Cardiomyopathy: Does the Type of Diabetes Matter? Int J Mol Sci. 2016 Dec 18;17(12) pii: E2136.

12. An D., Rodrigues B. Role of changes in cardiac metabolism in development of diabetic cardiomyopathy. Am J Physiol Heart Circ Physiol. 291 2006:H1489-H1506.

13. Giacco F, Brownlee M. Oxidative stress and diabetic complications. Circ Res. 2010;107(9):1058-70.

14. Bergerot C, Davidsen ES, Amaz $C$ et al. Diastolic function deterioration in type 2 diabetes mellitus: predictive factors over a 3-year follow-up. Eur Heart J Cardiovasc Imaging. 2017 Feb 6. doi: 10.1093/ehjci/jew331.

15. Fontes-Carvalho R, Ladeiras-Lopes R, Bettencourt $P$ et al. Diastolic dysfunction in the diabetic continuum: association with insulin resistance, metabolic syndrome and type 2 diabetes. Cardiovasc Diabetol. 2015 Jan 13;14:4. doi: 10.1186/ s12933-014-0168-x.

16. Paneni F, Gregori M, Tocci G et al. Do diabetes, metabolic syndrome or their association equally affect biventricular function? A tissue Doppler study. Hypertens Res. 2013 Jan;36(1):36-42.

17. Ofstad AP, Urheim S, Dalen $\mathrm{H}$ et al. Identification of a definite diabetic cardiomyopathy in type 2 diabetes by comprehensive echocardiographic evaluation: A cross-sectional comparison with non-diabetic weight-matched controls. J Diabetes. 2015 Nov; $7(6): 779-90$.

18. De Jong KA, Czeczor JK, Sithara S et al. Obesity and type 2 diabetes have additive effects on left ventricular remodelling in normotensive patients-a cross sectional study. Cardiovasc Diabetol. 2017 Feb 8;16(1):21.

19. Jørgensen PG, Jensen MT, Mogelvang R et al. Abnormal echocardiography in patients with type 2 diabetes and relation to symptoms and clinical characteristics. Diab Vasc Dis Res. 2016 Sep;13(5):321-30.

20. Rossi A, Zoppini G, Benfari G et al. Mitral Regurgitation and Increased Risk of All-Cause and Cardiovascular Mortality in Patients with Type 2 Diabetes. Am J Med. 2017 Jan;130(1):70-76.e1.

21. Blomstrand $P$, Engvall $M$, Festin $K$ et al. Left ventricular diastolic function, assessed by echocardiography and tissue Doppler imaging, is a strong predictor of cardiovascular events, superior to global left ventricular longitudinal strain, in patients with type 2 diabetes. Eur Heart J Cardiovasc Imaging. 2015;16(9):1000-7.

22. Naqvi TZ, Lee MS. Carotid intima-media thickness and plaque in cardiovascular risk assessment. JACC Cardiovasc Imaging. 2014;7(10):1025-38.
23. Niu L, Zhang Y, Qian M et al. Impact of multiple cardiovascular risk factors on carotid intima-media thickness and elasticity. PLoS One. 2013 2;8(7):e67809.

24. Baldassarre D, Hamsten A, Veglia F et al. IMPROVE Study Group. Measurements of carotid intima-media thickness and of interadventitia common carotid diameter improve prediction of cardiovascular events: results of the IMPROVE (Carotid Intima Media Thickness [IMT] and IMTProgression as Predictors of Vascular Events in a High Risk European Population) study. J Am Coll Cardiol. 2012 Oct 16;60(16):1489-99.

25. Plasencia Martínez JM, Garcia Santos JM, Paredes Martinez ML, Pastor AM. Carotid intima-media thickness and hemodynamic parameters: reproducibility of manual measurements with Doppler ultrasound. Med Ultrason. 2015;17(2):167-74.

26. Touboul PJ, Hennerici MG, Meairs $S$ et al. Mannheim carotid intima-media thickness and plaque consensus (2004-20062011). An update on behalf of the advisory board of the 3rd, 4th and 5th watching the risk symposia, at the 13th, 15th and 20th European Stroke Conferences, Mannheim, Germany, 2004, Brussels, Belgium, 2006, and Hamburg, Germany, 2011. Cerebrovasc Dis. 2012;34(4):290-6.

27. Gállego Pérez-Larraya J, Irimia P, Martínez-Vila E et al. The influence of obesity on the assessment of carotid intima-media thickness. J Clin Ultrasound. 2012 Oct;40(8):479-85.

28. Badeǐnikova KK, Mazaev AP, Toguzova ZA et al. [Detection of early markers of atherosclerosis in men with various levels of risk of cardiovascular complications]. Kardiologiia. 2014;54(6):35-9.

29. Silvestrini M, Altamura $C$, Cerqua $R$ et al. Ultrasonographic markers of vascular risk in patients with asymptomatic carotid stenosis. J Cereb Blood Flow Metab. 2013;33(4):619-24.

30. Lubas A, Kade G, Niemczyk S. Renal resistive index as a marker of vascular damage in cardiovascular diseases. Int Urol Nephrol. 2014;46(2):395-402.

31. Kawai T, Kamide K, Onishi M et al. Usefulness of the resistive index in renal Doppler ultrasonography as an indicator of vascular damage in patients with risks of atherosclerosis. Nephrol Dial Transplant. 2011;26(10):3256-62.

32. Bruno RM, Daghini E, Landini L, et al. Dynamic evaluation of renal resistive index in normoalbuminuric patients with newly diagnosed hypertension or type 2 diabetes. Diabetologia. 2011;54:2430-2439.

33. Mancini M, Masulli M, Liuzzi R et al. Renal duplex sonographic evaluation of type 2 diabetic patients. J Ultrasound Med. 2013;32(6):1033-40.

34. Maclsaac RJ, Thomas MC, Panagiotopoulos S et al. Association between intrarenal arterial resistance and diastolic dysfunction in type 2 diabetes. Cardiovasc Diabetol. 2008 23;7:15. doi: 10.1186/1475-2840-7-15.

35. Kinouchi M, Aihara K, Fujinaka $\mathrm{Y}$ et al. Diabetic conditions differentially affect the endothelial function, arterial stiffness and carotid atherosclerosis. J Atheroscler Thromb. 2014;21(5):486-500.

36. Staub D, Meyerhans A, Bundi B et al. Prediction of cardiovascular morbidity and mortality: comparison of the internal carotid artery resistive index with the common carotid artery intima-media thickness. Stroke. 2006;37(3):800-5.

37. Chung H, Jung YH, Kim KH et al. Carotid Artery EndDiastolic Velocity and Future Cerebro-Cardiovascular Events in Asymptomatic High Risk Patients. Korean Circ J. 2016;46(1):72-8. 